\title{
A reconstruction technique for long-range stimulated Brillouin scattering distributed fibre-optic sensors: experimental results
}

\author{
Aldo Minardo ${ }^{1,2}$, Romeo Bernini ${ }^{2,3}$, Luigi Zeni ${ }^{1,2}$, Luc Thevenaz ${ }^{4}$ \\ and Fabien Briffod ${ }^{5}$ \\ ${ }^{1}$ Dipartimento di Ingegneria dell'Informazione, Seconda Università di Napoli, Via Roma, 29, \\ 81031 Aversa, Italy \\ ${ }^{2}$ INFM, Naples, Italy \\ ${ }^{3}$ Istituto per il Rilevamento Elettromagnetico dell'Ambiente, Consiglio Nazionale delle \\ Ricerche, Via Diocleziano, 328-80124 Naples, Italy \\ ${ }^{4}$ EPFL, Swiss Federal Institute of Technology, Laboratory of Nanophotonics and Metrology, \\ 1015 Lausanne, Switzerland \\ ${ }^{5}$ OMNISENS SA, Parc Scientifique d'Ecublens, 1015 Lausanne, Switzerland \\ E-mail: zeni@unina.it
}

Received 20 September 2004, in final form 6 December 2004

Published 24 February 2005

Online at stacks.iop.org/MST/16/900

\begin{abstract}
The experimental validation of a numerical technique for temperature/strain profile reconstruction based on Brillouin optical-fibre time-domain analysis (BOTDA) sensors is presented. In this approach, we search directly for the Brillouin frequency shift profile along the fibre that matches the measured data. The algorithm is based on a harmonic expansion of the unknown profile, whose coefficients are determined by means of a multidimensional minimization. Experimental measurements have been carried out in order to reveal the influence of nonlocalities in Brillouin measurements, and to prove the capability of the proposed algorithm to compensate for these effects.
\end{abstract}

Keywords: Brillouin scattering, optical fibre sensors, distributed temperature sensors, distributed strain sensors

(Some figures in this article are in colour only in the electronic version)

\section{Introduction}

Stimulated Brillouin scattering (SBS) is a nonlinear effect that places a limit on the capabilities of the optical fibres, mainly in terms of the maximum launched optical power [1]. On the other hand, SBS can be advantageously utilized for distributed strain and temperature measurements.

The use of SBS for distributed fibre measurements was demonstrated by Horiguchi and Tateda in 1989 [2]. They used counterpropagating pulsed pump wave and CW Stokes probe wave and estimated the fibre loss from the amplification of the Stokes wave by SBS. Horiguchi and Tateda also carried out a first theoretical investigation of this method, named Brillouin optical-fibre time-domain analysis (BOTDA) [3].
In a BOTDA configuration, two counterpropagating lightwaves, at different frequencies, interact with an acoustic wave. The process of interaction between these waves is characterized by the Brillouin gain coefficient $g(z, v)$, where $z$ is the abscissa along the fibre and $v$ is the frequency difference between the two lightwaves. The gain is maximum for $v=v_{\mathrm{B}}$, named Brillouin frequency shift, which can be determined by monitoring the dependence of the power transfer from one lightwave to the other on $v$. Since $v_{\mathrm{B}}$ changes linearly with both temperature and strain, these parameters can be extracted by the measurement of $v_{\mathrm{B}}$. Spatial information is achieved by simultaneously using pulsed and counterpropagating $\mathrm{CW}$ light. The spatial resolution $\Delta z$ is equal to the pulse-CW light interaction length, that is half the pulse length. Two 


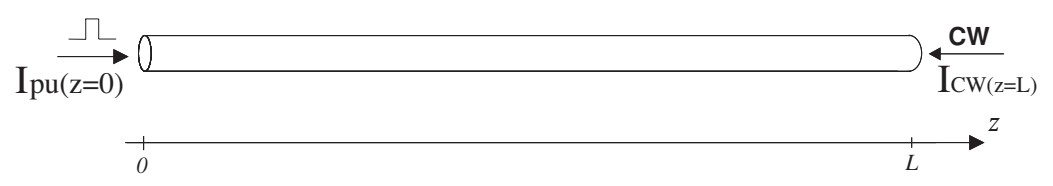

Figure 1. Stimulated Brillouin scattering interaction between a pulsed lightwave and a counterpropagating probe lightwave.

configurations are possible: the first occurs when the frequency of the CW lightwave is higher than that of the pulsed one, the power being transferred in this case from the $\mathrm{CW}$ light to the pulse, and the second occurs when the CW light has the lower frequency, now the power transfer is from the pulse to the $\mathrm{CW}$ light. The first configuration is referred to as the loss process since the CW light loses power; the second one as the gain process, because of the $\mathrm{CW}$ power gain.

The reconstruction of the Brillouin frequency shift profile along the fibre is usually made by evaluating the Brillouin gain/loss spectrum for each sensing section. Each spectrum is produced by measuring the intensity of the transmitted CW field while scanning the frequency offset between the two beams. At a specified section, the Brillouin frequency shift is evaluated by fitting a spectral shape, typically a Lorentzian, to the measured spectrum [4]. However, this approach relies on the assumption that a linear relationship exists between the Brillouin gain coefficient and Brillouin signals measured for each fibre section. As a matter of fact, the Brillouin signal arising from a certain location along the fibre is dependent even on the Brillouin interaction at every preceding section along the fibre itself, via the pulse intensity. Thus, the Brillouin signal is influenced by nonlocal effects, leading to systematic errors that increase with the sensing length and the involved optical powers $[5,6]$. Such a reduction in the accuracy of SBS reconstructions is particularly severe when considering long-range measurements. On the other hand, SBS-based sensors are of particular interest when long distances are to be monitored, as they can replace several point sensors. Limitations imposed by nonlocality, however, can be overcome by the use of more sophisticated signal processing techniques. A compensation technique was first proposed which relies on the recording of both $\mathrm{CW}$ and pulsed wave intensity changes [6]. However, in this case the experimental set-up has to be somehow complicated in comparison to the BOTDA basic configuration, since even the pulse energy emerging from the fibre needs to be measured for error compensation. An iterative technique for the reconstruction of Brillouin frequency shift profiles along an optical fibre, based on BOTDA measurements, has been recently proposed [7]. This approach, named the harmonic approach, is based on the decomposition of the unknown Brillouin frequency shift profile into a finite number of base harmonic functions. The coefficients of these harmonics are determined through a suitable numerical processing of the time-domain Brillouin gain/loss signals. Numerical simulations have proved the capability of the proposed algorithm to compensate for nonlocal effects, even when considering longer fibres and higher optical power levels.

In this paper, the capability of the harmonic algorithm to cope with nonlocal effects is experimentally demonstrated. The measurements employed for algorithm validation have been carried out by using the DiTeSt, a commercial instrument implementing a BOTDA configuration. The achieved results indicate that the proposed algorithm is able to fully compensate for nonlocal effects occurring along the fibre, both in Brillouin loss and Brillouin gain regimes.

In what follows, we first provide, in section 2, the theoretical model describing the SBS interaction in optical fibres. This model will be employed both for an understanding of nonlocal effects in time-domain SBS measurements, and for the definition of the proposed reconstruction technique. The latter will be described in section 3. Finally, an extensive set of experimental tests proving the effectiveness of our algorithm is presented in section 4 .

\section{Theoretical model}

SBS interaction between a pulsed pump laser beam and a counterpropagating $\mathrm{CW}$ probe beam can be described by using the steady-state coupled intensity SBS equations, as long as the pulse width is sufficiently longer than the phonon lifetime $(\approx 10 \mathrm{~ns})$ [8]. Assuming a fibre loss coefficient $\alpha$, and the input intensities $I_{\mathrm{pu}}(z=0)=I_{\mathrm{pu} 0}$ and $I_{\mathrm{CW}}(z=L)=I_{\mathrm{CWL}}$ (figure 1), the pulse intensity along the fibre can be calculated by using the perturbation model described in [9]

$$
I_{\mathrm{pu}}(z, v)=I_{\mathrm{pu} 0} \exp (-\alpha z) G(z, v)
$$

where the factor $G(z, v)$ is given by

$$
\begin{aligned}
& G(z, v)=\exp \left(\int_{0}^{z}-g_{\mathrm{B}}\left(z^{\prime}, v\right) I_{\mathrm{CWL}} \exp \left(-\alpha\left(L-z^{\prime}\right)\right) \mathrm{d} z^{\prime}\right) \\
& 0<z<L
\end{aligned}
$$

and represents the attenuation/amplification experienced by the pulsed beam due to Brillouin interaction. The Brillouin gain coefficient $g_{\mathrm{B}}(z, v)$ depends at each section $z$ on the local Brillouin frequency shift $\nu_{\mathrm{B}}(z)$ and the pump-probe frequency offset $v$, according to a spectral function, the so-called Brillouin gain spectrum (BGS), having typically a Lorentzian shape [8]. Moreover, the BGS peak, $g_{\mathrm{B} 0}$, depends at each section on the states of the polarization of the two beams, so that the Brillouin gain $g_{\mathrm{B}}$ may vary along the fibre even in the condition of a uniform Brillouin frequency shift profile. That is, we can write

$$
g_{\mathrm{B}}(\nu, z)=\frac{g_{\mathrm{B} 0}(z)}{1+\left(\frac{\nu-v_{\mathrm{B}}(z)}{\Delta \nu_{\mathrm{B}} / 2}\right)^{2}}=\frac{\gamma(z) g_{0}}{1+\left(\frac{\nu-\nu_{\mathrm{B}}(z)}{\Delta \nu_{\mathrm{B}} / 2}\right)^{2}}
$$

where $\Delta v_{\mathrm{B}}$ is the full width at half maximum (FWHM) BGS linewidth; $g_{0}$ is the peak gain, $\gamma$ is a polarization factor, which accounts for the dependence of gain on the polarization of the two beams [10]. The sign of $g_{\mathrm{B}}$ has to be chosen positive in the case of Brillouin gain process and negative in the case of 
Brillouin loss process. The pulse intensity profile permits us to calculate the temporal changes in the $\mathrm{CW}$ probe intensity received at $z=0$, for each instant $t$ following the launch of the pulse and for each pump-probe frequency offset $v$

$$
\begin{aligned}
& \Delta I_{\mathrm{CW}}(t, v) \equiv\left|I_{\mathrm{cW}}(z=0, t, v)-I_{\mathrm{CWL}} \exp (-\alpha L)\right| \\
&=I_{\mathrm{CWL}} \exp (-\alpha L) \\
& \times\left\{\exp \left[\int_{t v_{\mathrm{g}} / 2+\Delta z}^{t v_{\mathrm{g}} / 2}-g_{\mathrm{B}}\left(z^{\prime}, v\right) I_{\mathrm{pu}}\left(z^{\prime}, v\right) \mathrm{d} z^{\prime}\right]-1\right\} \\
& 0<t<2(L-\Delta z) / v_{\mathrm{g}} .
\end{aligned}
$$

In equation (4), $\Delta z$ represents the pump-probe interaction length and is given by $W / 2 v_{\mathrm{g}}, W$ being the pump pulse width and $v_{\mathrm{g}}$ the group velocity in the fibre.

The above equations can be used to study the errors induced by nonlocal effects. When the gain factor $G(z, v)$, defined in equation (2), is nearly equal to unity for any pump-probe frequency offset $v$ and any section $z$, amplification/depletion induced by the $\mathrm{CW}$ beam on the pulse intensity is negligible, so that pulse intensity changes are mainly due to fibre loss. As pulse attenuation due to fibre loss does not depend on the frequency offset $v$, we can observe from equation (4) that any generic Brillouin signal spectrum $\Delta I_{\mathrm{CW}}\left(2 \bar{z} / v_{\mathrm{g}}, v\right)$ will present the same spectral features as the Brillouin gain coefficient $g_{\mathrm{B}}(\bar{z}, v)$ at the considered section. Typically, $\Delta I_{\mathrm{CW}}\left(2 \bar{z} / v_{\mathrm{g}}, v\right)$ presents a Lorentzian shape with its centre located at the Brillouin frequency shift $v_{\mathrm{B}}(\bar{z})$ [8]. At most, the exponential function applied to $g_{\mathrm{B}}$ may induce a narrowing of the recorded spectrum with respect to the intrinsic Brillouin gain linewidth $\Delta v_{\mathrm{B}}$, with increasing pulse intensities [11]. This situation radically changes when $G<$ 1 (Brillouin gain) or $G>1$ (Brillouin loss). In this case, the dependence of the pulse intensity $I_{\mathrm{pu}}$ on the frequency offset $v$ induces a distortion on the recorded Brillouin signal spectrum $\Delta I_{\mathrm{CW}}\left(2 \bar{z} / v_{\mathrm{g}}, v\right)$. The most harmful effect of such distortion is a deviation of the peak frequency of the recorded spectrum from the local Brillouin frequency shift $v_{\mathrm{B}}(\bar{z})$. This means that the Brillouin frequency shift estimated at the generic section $\bar{z}$ will differ from the actual Brillouin frequency shift $v_{\mathrm{B}}(\bar{z})$, resulting in a systematic error in temperature/strain evaluation [5-7]. The amount of such errors depends on the pulse intensity profile, which in turn is dependent on the whole Brillouin frequency shift distribution along the fibre and the input power levels.

\section{Description of the algorithm}

In this section, we briefly report the operation of the harmonic algorithm for Brillouin frequency shift reconstruction. A detailed description of the harmonic algorithm can be found in [7]. The harmonic approach makes use of equation (4) in order to evaluate the actual Brillouin interaction related to any Brillouin frequency shift distribution, provided that the BGS functional shape is known. To this aim, different techniques can be used in order to provide an accurate characterization of the fibre acting as a sensor $[12,13]$.

The profile to be reconstructed may be either a temperature or a strain profile, depending on the effect that induces the changes in Brillouin frequency shift along the fibre. Here, we generally refer to a Brillouin frequency shift profile. This can be converted afterwards into a temperature or strain profile by using the suitable transduction factor.

The proposed algorithm is based on the expansion of the unknown Brillouin frequency shift profile by using a suitable space of base functions. In order to deal with a reduced set of unknown parameters, we represent the profile as a truncated Fourier series:

$$
\begin{aligned}
f_{\mathrm{B}}(z) & =\sum_{n=-N}^{N} f_{n} \mathrm{e}^{\mathrm{j} n \omega_{f} z}=f_{0}+2 \operatorname{Re}\left(\sum_{n=1}^{N} f_{n} \mathrm{e}^{\mathrm{j} n \omega_{f} z}\right) \\
0 & \leqslant z \leqslant L
\end{aligned}
$$

where $L$ is the length of the fibre, $\omega_{f}=2 \pi / L$ is the fundamental spatial frequency and $f=\left\{f_{n}\right\}_{n=0}^{N}$ is the sequence of unknown complex coefficients. The above profile stands for the difference between the actual Brillouin frequency shift along the fibre and a reference Brillouin frequency shift profile, that is

$$
v_{\mathrm{B}}(z)=v_{\mathrm{Bref}}(z)+f_{\mathrm{B}}(z) .
$$

It will be argued later that a good choice for $v_{\mathrm{Bref}}(z)$ is the profile resulting for the classical reconstruction technique.

The algorithm searches for the complex coefficients $f_{n}$ of expansion (5), which minimize a cost function defined as the square norm, in the $t$ space, of the difference between the model signals and the measured signals, that is

$F(\underline{f})=\sum_{i=1}^{M}\left\|\Delta I_{\mathrm{CW}(\text { Model })}\left(\underline{f}, t, v_{i}\right)-\Delta I_{\mathrm{CW} \text { (Measured) }}\left(t, v_{i}\right)\right\|^{2}$

$M$ being the number of pump-probe frequency shifts $v_{i}$ used for the measurements. Hence, the algorithm runs by simultaneously processing all the Brillouin signals related to the different frequency shifts.

The algorithm starts by numerically evaluating $I_{\mathrm{pu}}$, for the gain distribution related to the reference Brillouin frequency shift profile $v_{\mathrm{Bref}}(z)$. Hence, the integral in equation (2) is solved for the gain distribution related to $v_{\mathrm{B}}(z)=v_{\mathrm{Bref}}(z)$. Then, the Brillouin signals related to the specified Brillouin frequency shift profile can be obtained by iteratively solving equation (4):

$$
\begin{aligned}
& \Delta I_{\mathrm{CW}}\left(t, v_{i}\right)=I_{\mathrm{CWL}} \exp (-\alpha L) \\
& \quad \times\left\{\exp \left[\int_{t v_{\mathrm{g}} / 2+\Delta z}^{t v_{\mathrm{g}} / 2}-g_{\mathrm{B}}\left(z^{\prime}, v_{i}\right) I_{\mathrm{pu}(\mathrm{ref})}\left(z^{\prime}, v_{i}\right) \mathrm{d} z^{\prime}\right]-1\right\} \\
& i=1, \ldots, M \quad 0<t<2(L-\Delta z) / v_{\mathrm{g}}
\end{aligned}
$$

The number $N$ of harmonics employed for the representation of $f_{\mathrm{B}}$ is not fixed a priori. Instead, the number of searched unknown coefficients is progressively increased during the minimization. This is also a useful way to avoid problems related to nonlocal minima which may occur in nonlinear minimization procedures [14].

The number of harmonic components for $f_{\mathrm{B}}(z)$ is initially set to zero, i.e. only the direct component is searched for. When a minimum for the function $F$ is achieved, the number of the harmonics is increased and the minimization algorithm starts again. The harmonic coefficients determined after each step 
are used as the starting guess for the next one and, at each minimization step, the profile estimated with $n$ harmonics is compared to the profile estimated with $(n-1)$ harmonics. The expansion is arrested when the norm of the estimated Brillouin frequency shift distribution differs, with respect to the norm of the previous one, by a quantity less than $1 \%$. Once the convergence condition over $N_{1}$ harmonics is achieved, the algorithm updates the pulse intensity solutions by evaluating $I_{\mathrm{pu}}(z)$ related to the currently estimated profile. Thus, a new minimization procedure is started by using the same number $N_{1}$ of harmonics, but evaluating the model Brillouin signals in $F$ with the new calculated $I_{\mathrm{pu}}(z)$ solutions:

$$
\begin{aligned}
& \Delta I_{\mathrm{CW}}\left(t, v_{i}\right)=I_{\mathrm{CWL}} \exp (-\alpha L) \\
& \quad \times\left\{\exp \left[\int_{t v_{\mathrm{g}} / 2+\Delta z}^{t v_{\mathrm{g}} / 2}-g_{\mathrm{B}}\left(z^{\prime}, v_{i}\right) I_{\text {pu(updated })}\left(z^{\prime}, v_{i}\right) \mathrm{d} z^{\prime}\right]-1\right\} \\
& i=1, \ldots, M \quad 0<t<2(L-\Delta z) / v_{\mathrm{g}} .
\end{aligned}
$$

At this point, the harmonic expansion starts again, by using the new values of pump intensity ( $\left.I_{\text {pu(updated) }}\right)$ and the $N_{1}$ harmonic coefficients so far estimated as a starting guess. Once a new convergence condition over the number of harmonics $N_{2}>N_{1}$ is reached, the pump intensity related to this new reconstruction is evaluated again. Similarly, a new minimum is then searched, by using here $N_{2}$ harmonics and the last updated pump intensity values for the calculation of the Brillouin signals.

The final convergence condition is evaluated by comparing the profile reconstructions obtained after each expansion stage, i.e. the profiles estimated whenever the convergence condition over the number of harmonics is fulfilled. The final Brillouin frequency shift profile estimate is considered accurate when its norm differs from that of the previous one by a quantity less than $1 \%$.

For a better understanding of the algorithm, we summarize its operation by means of a flow chart showing the different steps performed (see figure 2).

\section{Experimental results}

In this section, we present some examples aimed at experimentally validating the harmonic reconstruction technique. A set of measurements has been carried out by using the DiTeSt, a portable instrument manufactured by the Swiss company OMNISENS. The instrument permits us to perform distributed temperature and strain measurements along an optical fibre, by implementing a BOTDA configuration featuring a laser source emitting at $\lambda=1.55 \mu \mathrm{m}$. Although the instrument may directly provide the reconstructed temperature/strain profiles by employing a standard direct spectroscopic approach, the BOTDA signals measured by the DiTeSt have been collected and processed on a separate PC unit, in order to compare the reconstructions performed with the classical and the novel harmonic approach.

BOTDA measurements have been performed both in Brillouin gain and Brillouin loss regimes. The choice between the two processes could be made by properly setting the DiTeSt operation mode. An external EDFA has been employed in order to boost the probe injected power. The probe injected power could be varied by acting on an external variable

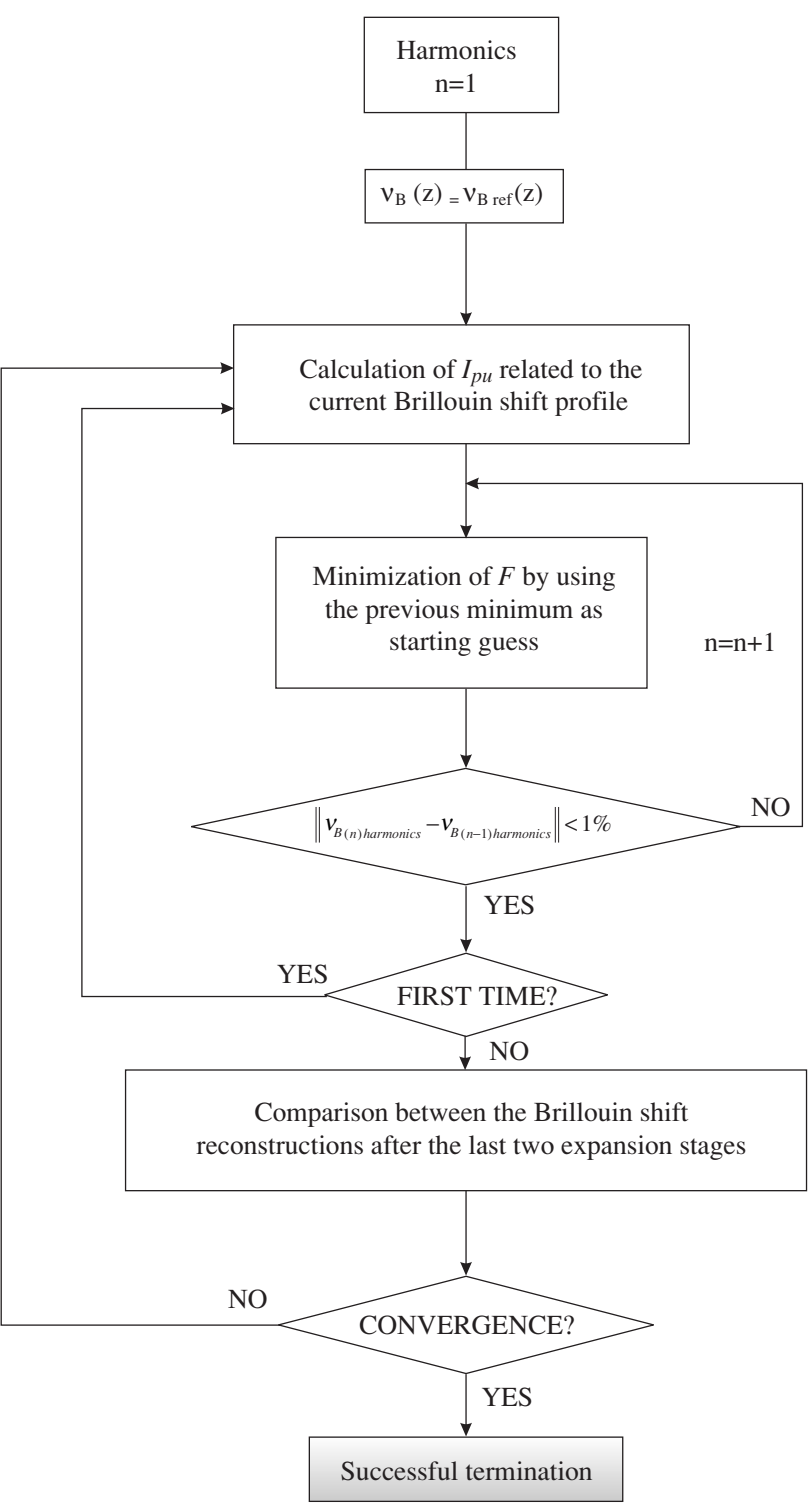

Figure 2. Flow chart of the reconstruction algorithm.

attenuator placed after the EDFA, providing up to $30 \mathrm{~dB}$ optical power attenuation. The possibility to vary the injected probe power permitted the analysis of the influence of this parameter on the accuracy of the Brillouin frequency shift reconstructions when applying the classical reconstruction approach.

The pulse width was set to $W=30 \mathrm{~ns}$ throughout the measurements, corresponding to a $3 \mathrm{~m}$ spatial resolution, whereas the sampling step for the acquired temporal traces was set to $20 \mathrm{~ns}$. Note that the finite spatial resolution poses a lower limit on the spatial wavelength, which corresponds to the highest harmonic component employed for the representation of the Brillouin frequency shift profile (see equation (5)). The peak power of the injected optical pulses was set to two different values depending on the kind of BOTDA process involved: we set $P_{\mathrm{P} 0}=35 \mathrm{~mW}$ in the case of the Brillouin loss process, and $P_{\mathrm{P} 0}=370 \mathrm{~mW}$ in the case of the Brillouin gain process. The reason for this difference lies in the fact that the pulse is amplified during propagation in the Brillouin loss, while being depleted in Brillouin gain. The extent of 
such an amplification/depletion depends on the fibre length and the intensity of the counterpropagating probe signal. The conditions set for our experimental tests resulted in a pulse intensity gain factor $G$ due to SBS interaction up to about 10 in Brillouin gain, and down to about 0.1 in Brillouin loss, so that a careful choice of the injected pulse power was of importance for a correct acquisition of the Brillouin signals. The maximum input pulse power in the Brillouin loss process (where the pulse is amplified by SBS) was limited by the onset of other nonlinear effects occurring when the pulse power exceeds some definite thresholds. Instead, the input pulse power in Brillouin gain process (where the pulse is depleted by SBS interaction) was set to an order of magnitude higher value in order to assure a sufficient pump power throughout the interaction length.

The DiTeSt also features a polarization scrambler, so that the Brillouin gain peak can be considered as uniform along the fibre, its value being equal to half the maximum gain occurring when the polarization states of pump and probe beams are kept parallel along the interaction length, i.e. $\gamma(z)=1 / 2$ in equation (3) [10]. Such an occurrence permitted us, when performing the harmonic reconstruction, to relate any changes in $g_{\mathrm{B}}$ along the fibre to Brillouin frequency shift changes only, as the Brillouin gain peak is a constant.

The fibre chosen for the measurements was a $7 \mathrm{~km}$ long single-mode dispersion-shifted fibre (DSF), which was wound around a spool of about $50 \mathrm{~cm}$ diameter, and was not subjected to external temperature or strain changes. The pump-probe frequency offset has been stepped from $10410 \mathrm{MHz}$ to $10610 \mathrm{MHz}$ with a $2 \mathrm{MHz}$ uniform step.

A first analysis has been carried out in order to study the influence of the probe power on the accuracy of the reconstructions when applying the classical direct approach. With this aim, the Brillouin frequency shift distribution of the fibre under test has been determined by applying the classical technique for a range of input probe powers, both in Brillouin loss and Brillouin gain.

The first measurements have been performed in the Brillouin loss process, while setting an input optical probe power $P_{\mathrm{CWL}}=200 \mu \mathrm{W}$ and $P_{\mathrm{CWL}}=500 \mu \mathrm{W}$. The reconstructed Brillouin frequency shift profiles for the two different probe power levels are shown in figure 3. The two profiles appear very similar, as confirmed by a root mean square (RMS) deviation between the two profiles as low as $0.45 \mathrm{MHz}$. Such a circumstance suggests that no appreciable nonlocality effect occurs for input probe powers up to $500 \mu \mathrm{W}$. As a consequence, any of the two above shown profiles can be adopted as the reference profile, i.e. the 'actual' profile. As we can see, a difference up to $15 \mathrm{MHz}$ exists between the Brillouin frequency shifts in the initial and final regions of the sensing fibre. These changes are due to the strain induced by the spool winding, a Brillouin frequency shift variation of $15 \mathrm{MHz}$ corresponding to an axial strain of about $300 \mu \varepsilon$.

The influence of an higher input probe power has been analysed by increasing the injected probe power up to $4700 \mu \mathrm{W}$, while keeping constant the input pulse power to $P_{\mathrm{P} 0}=35 \mathrm{~mW}$. The presence of nonlocal effects induced by pulse amplification is the reason for the difference among the different profiles shown in figure 4. Nonlocal effects are shown to induce an error in the Brillouin frequency shift

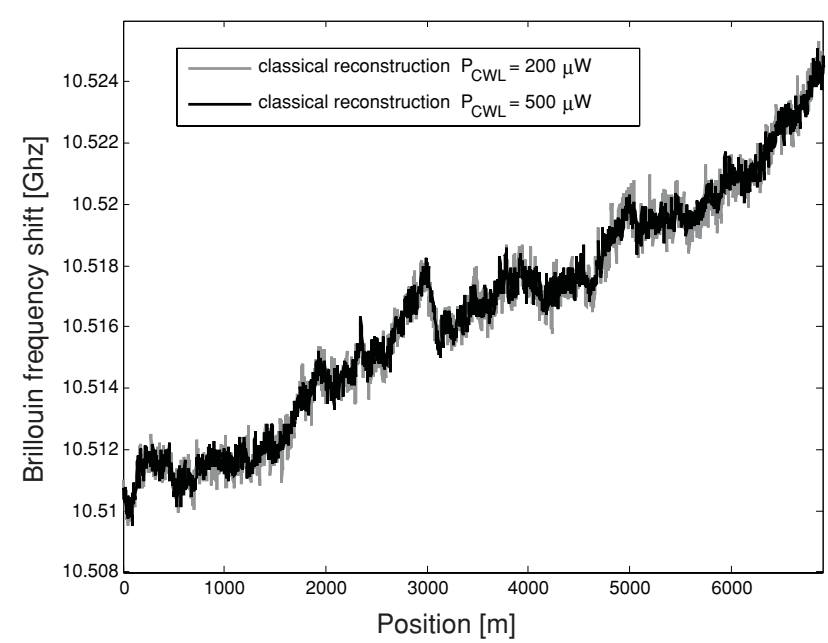

Figure 3. Reconstruction of the Brillouin frequency shift profile along a $7 \mathrm{~km}$ long fibre by processing BOTDA loss measurements using the classical approach. The input optical powers are $P_{\mathrm{P} 0}=$ $35 \mathrm{~mW}$ and $P_{\mathrm{CWL}}=200 \mu \mathrm{W}$ (grey line), $P_{\mathrm{CWL}}=500 \mu \mathrm{W}$ (black line).

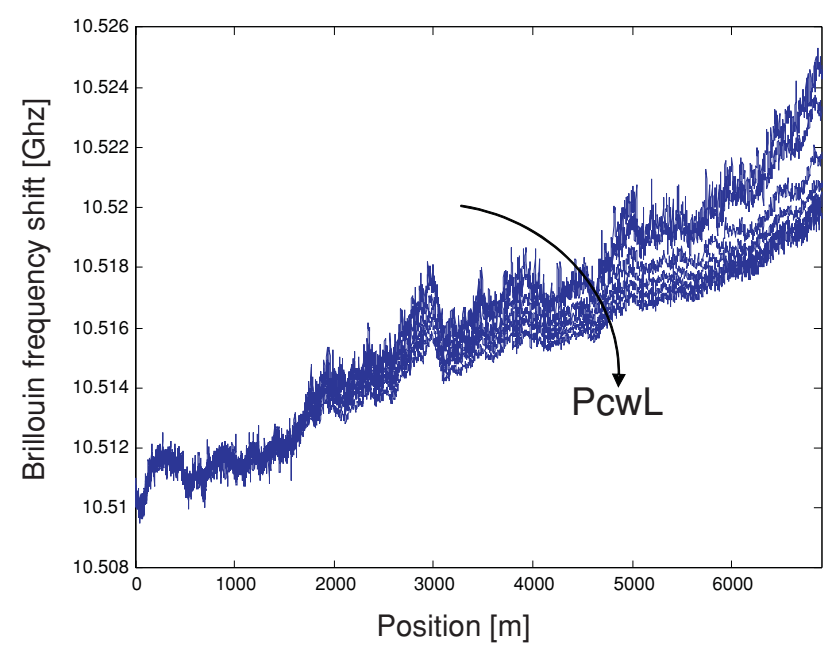

Figure 4. Reconstruction of the Brillouin frequency shift profile along a $7 \mathrm{~km}$ long fibre by processing BOTDA loss measurements using the classical approach. The input pulse power is $P_{\mathrm{P} 0}=$ $35 \mathrm{~mW}$. The input probe powers are $200 \mu \mathrm{W}, 1015 \mu \mathrm{W}, 2135 \mu \mathrm{W}$, $3080 \mu \mathrm{W}, 4000 \mu \mathrm{W}, 4700 \mu \mathrm{W}$.

determination as high as $5 \mathrm{MHz}$, when considering the last region of the fibre and the higher input probe power. In particular, a significant deviation from the reference profile (the one measured with an input probe power of $200 \mu \mathrm{W}$ ) occurs when the input probe power exceeds $1000 \mu \mathrm{W}$. Such a deviation increases along the fibre and can be appreciated after the first two kilometres of fibre length.

A set of BOTDA measurements has then been performed in the Brillouin gain regime, for a range of input probe powers. As stated before, the input pulse power in the Brillouin gain measurements was set to $P_{\mathrm{P} 0}=370 \mathrm{~mW}$. The results are summarized in figure 5 . The error induced by nonlocal effects in the final region is shown to be as high as $35 \mathrm{MHz}$, against the maximum value of $5 \mathrm{MHz}$ observed for Brillouin loss measurements. Such a difference is mainly due to the different 


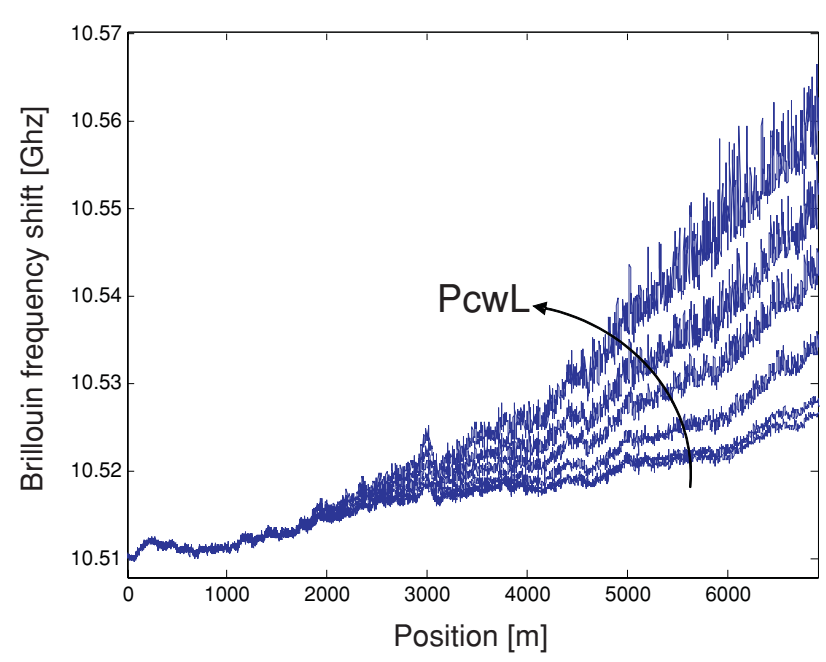

Figure 5. Reconstruction of the Brillouin frequency shift profile along a $7 \mathrm{~km}$ long fibre by processing BOTDA gain measurements using the classical approach. The input pulse power is $P_{\mathrm{P} 0}=$ $370 \mathrm{~mW}$. The input probe powers are $530 \mu \mathrm{W}, 1000 \mu \mathrm{W}, 2045 \mu \mathrm{W}$, $3040 \mu \mathrm{W}, 4035 \mu \mathrm{W}, 5000 \mu \mathrm{W}$.

distortion induced by nonlocality in the two regimes. In fact, Brillouin spectra are strongly attenuated in the Brillouin gain regime at the frequencies where the gain factor $G(z, v)$ of equation (2) is higher than unity, due to pulse depletion. Instead, pulse amplification occurring in the Brillouin loss process enhances the spectra at the frequencies where $G(z, v)$ is less than unity. For the analysed Brillouin frequency shift profile, the spectral distortion occurring in the Brillouin gain regime leads to the larger peak frequency deviations from the actual Brillouin frequency shifts. The analysis of figure 4 also reveals that, due to the strong pulse depletion induced by SBS, the use of a higher input probe power in the Brillouin gain regime results in a significant signal-to-noise ratio (SNR) measurement reduction along the fibre and hence in a noisier reconstruction of the Brillouin frequency shift profile in the final region.

The harmonic reconstruction algorithm, described in section 3, has been employed for the processing of the BOTDA measurements provided by the DiTeSt. With this aim, the reconstruction provided by the classical technique has been used each time as the starting profile for our algorithm. This choice is justified by the consideration that, in this fashion, only the errors induced by nonlocal effects are to be compensated by the term $f_{\mathrm{B}}(z)$ in equation (6). As these errors are spatially smoother than the actual profile $v_{\mathrm{B}}(z)$, a reduced number of harmonic components is required for a correct representation of the function $f_{\mathrm{B}}(z)$, allowing the computational time for our algorithm to be decreased.

The number of processed signals has been restricted in order to further reduce the computational effort. In particular, only the measurements corresponding to a pump-probe frequency offset ranging from $10480 \mathrm{MHz}$ to $10550 \mathrm{MHz}$ have been employed for the harmonic technique processing, thus $M$ (number of processed signals) $=36$. It must be pointed out that the proposed technique does not impose a limit on the spectrum range covered by the measurements. When dealing with a range of a few hundred $\mathrm{MHz}$, the number of processed

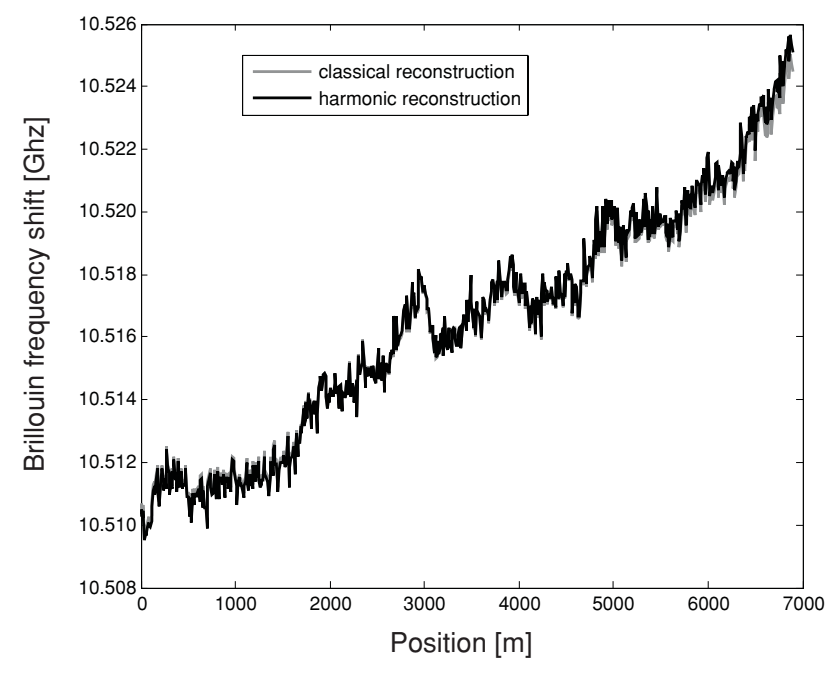

Figure 6. Reconstruction of the Brillouin frequency shift profile along a $7 \mathrm{~km}$ long fibre by processing BOTDA loss measurements using the classical approach (grey line) and the harmonic approach (black line). The input optical powers are $P_{\mathrm{P} 0}=35 \mathrm{~mW}$ and $P_{\mathrm{CWL}}=200 \mu \mathrm{W}$.

signals can be kept low by increasing the frequency step between the different measurements. In fact, we recall that the harmonic reconstruction does not rely on the sampling of the Brillouin gain spectrum at each fibre section, but is performed by processing simultaneously all the Brillouin signals related to the different frequency shifts.

As a first test case, the BOTDA measurements performed with $P_{\mathrm{CWL}}=200 \mu \mathrm{W}$ (reference profile) have been processed by our algorithm. The result is shown in figure 6 . It is apparent that the two reconstructions match very well, as expected because of the low input probe power used for this measurement. This demonstrates that, when no appreciable nonlocal effects occur, the Brillouin frequency shift reconstruction provided by our algorithm agrees with the one provided by the classical technique. The RMS deviation between the two reconstructions shown in figure 6 , is only $0.17 \mathrm{MHz}$.

The measurement performed in the Brillouin loss regime with the highest input probe power $\left(P_{\mathrm{CWL}}=4700 \mu \mathrm{W}\right)$ has been considered next. Classical and harmonic reconstructions are shown in figure 7 . The harmonic technique is shown to be able to fully compensate for nonlocal effects, matching very well with the reference profile. RMS deviation between reference profile and harmonic reconstruction is $0.58 \mathrm{MHz}$. Such a result demonstrates that the harmonic technique allows us to increase the input probe power (and then the SNR when operating in the Brillouin loss regime), without incurring the systematic errors induced by nonlocality.

Even though the results presented in figures 4 and 5 suggest that Brillouin loss operation is preferable to Brillouin gain in terms of signal-to-noise ratio, we have also tested the harmonic approach with Brillouin gain measurements. A first test has been performed for the input probe power $P_{\mathrm{CWL}}=2045 \mu \mathrm{W}$. The usual quantities are plotted in figure 8 . Still, an excellent compensation of nonlocal effects is provided by the harmonic algorithm, leading to an RMS deviation from the reference profile as low as $0.75 \mathrm{MHz}$. 


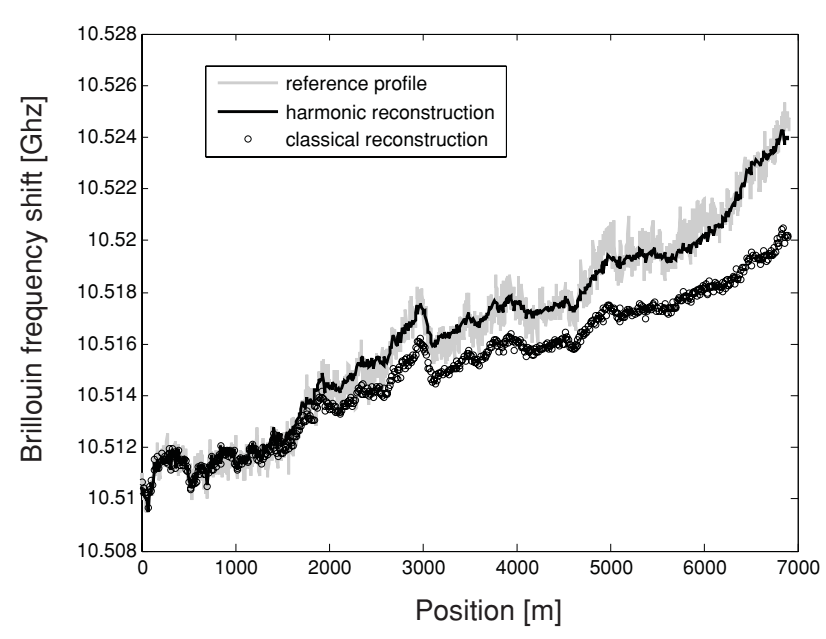

Figure 7. Reconstruction of the Brillouin frequency shift profile along a $7 \mathrm{~km}$ long fibre by processing BOTDA loss measurements using the classical approach (circles) and the harmonic approach (black line), compared to the reference profile (grey line). The input optical powers are $P_{\mathrm{P} 0}=35 \mathrm{~mW}$ and $P_{\mathrm{CWL}}=4700 \mu \mathrm{W}$.

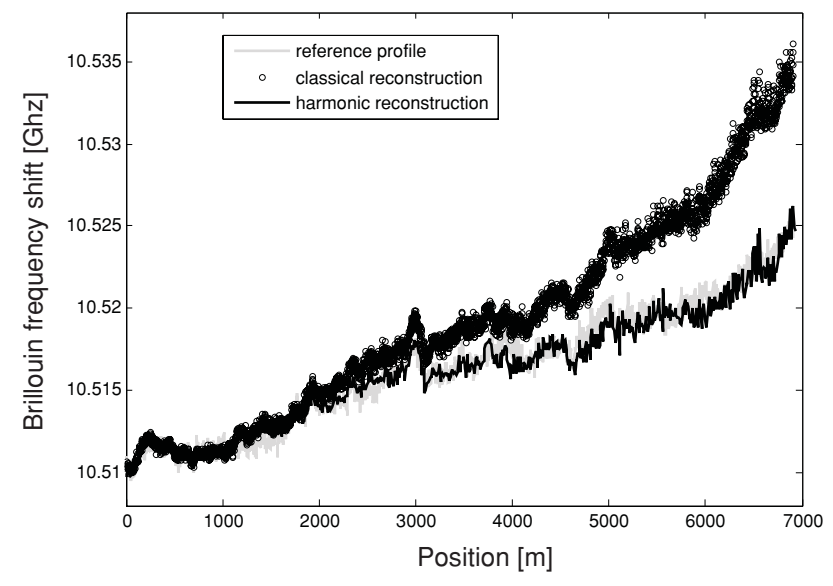

Figure 8. Reconstruction of the Brillouin frequency shift profile along a $7 \mathrm{~km}$ long fibre by processing BOTDA gain measurements using the classical approach (circles) and the harmonic approach (black line), compared to the reference profile (grey line). The input optical powers are $P_{\mathrm{P} 0}=370 \mathrm{~mW}$ and $P_{\mathrm{CWL}}=2045 \mu \mathrm{W}$.

The case of input probe power $P_{\mathrm{CWL}}=5000 \mu \mathrm{W}$ is considered next, the results being shown in figure 8 . While nonlocal effect compensation is still achieved, the harmonic reconstruction appears noisier with respect to the previous examples, the RMS deviation from the reference profile being equal to $1.69 \mathrm{MHz}$. This is due to the noisier classical reconstruction, which is employed as a starting profile in our algorithm. In fact, the choice of starting from the classical reconstruction, and then adding to it the calculated harmonic components, has the drawback that the high-frequency noise components present in the classical reconstruction also affect the harmonic reconstruction. This occurrence could be avoided by choosing a uniform profile as a starting guess when applying the harmonic approach. In fact, when starting from a uniform profile, the harmonic algorithm is able to perform an intrinsic filtering of the noise whose spectral content falls outside the range of the harmonics used for the reconstruction [7]. However, a much higher number of harmonic components

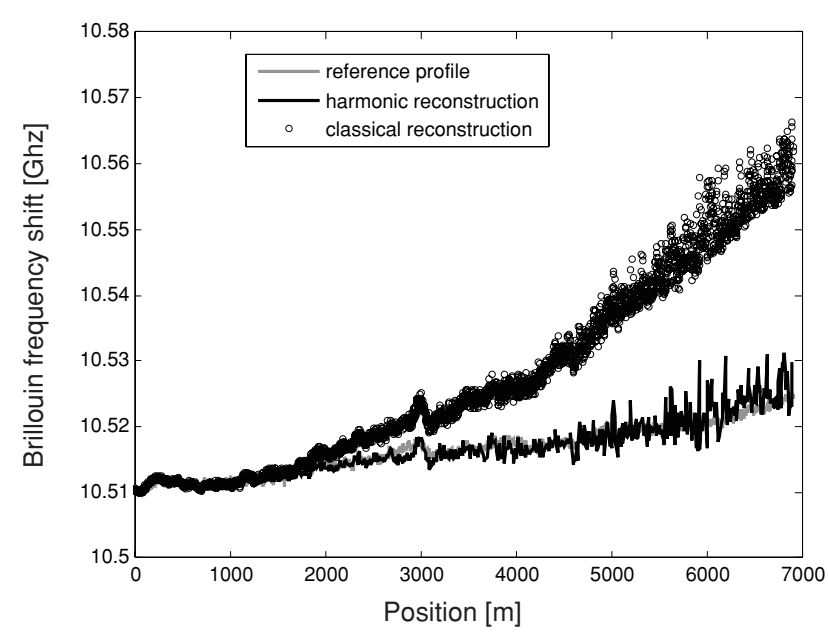

Figure 9. Reconstruction of the Brillouin frequency shift profile along a $7 \mathrm{~km}$ long fibre by processing BOTDA gain measurements using the classical approach (circles) and the harmonic approach (black line), compared to the reference profile (grey line). The input optical powers are $P_{\mathrm{P} 0}=370 \mathrm{~mW}$ and $P_{\mathrm{CWL}}=5000 \mu \mathrm{W}$.

would be required in this case to obtain a reconstruction with higher spatial resolution (i.e. the one imposed by the $30 \mathrm{~ns}$ pulse width). For example, a number of harmonic components greater than 2000 should be considered for a $7 \mathrm{~km}$ long fibre. Obviously, a lower number of harmonic components could still be considered when choosing the uniform profile as the starting guess, but only a low-pass version of the actual profile may be reconstructed in that case. By using the classical reconstruction as the starting guess, we only need to compensate for systematic errors induced by nonlocalities. These errors are spatially much smoother than the overall Brillouin shift profile, so that a number below 20 of harmonic components was sufficient to represent the term $f_{\mathrm{B}}(z)$ of equation (6), with a computational time of the order of $10 \mathrm{~min}$. The resulting harmonic reconstruction (see figure 9) is still much more accurate than classical reconstruction, the latter being affected by an RMS error of $15.8 \mathrm{MHz}$. We must emphasize that in the Brillouin gain regime, the input probe power must be kept relatively low in order to avoid a significant pump depletion. However, this last experiment was instrumental to demonstrate the capability of the proposed algorithm to compensate for nonlocal effects, independently of the power of the interacting beam and of the fibre length.

Further algorithm tests have been performed on the basis of a new set of BOTDA measurements, where a second $50 \mathrm{~m}$ long single-mode fibre has been connected to the end of the fibre considered so far. Such a measurement condition may occur when a first long fibre is used for remotely interrogating a shorter fibre, connected to the end of the first one. The reconstruction of the Brillouin frequency shift profile along the whole interaction length has been achieved with our algorithm by using the previously calculated profile over the $7 \mathrm{~km}$ long fibre, and then calculating the unknown profile over the final $50 \mathrm{~m}$ long region. A different set of BGS parameters has been considered for the two fibres, as they have been manufactured by different producers.

The first results refer to the case of Brillouin loss measurement with $P_{\mathrm{CWL}}=200 \mu \mathrm{W}$, and are shown in 


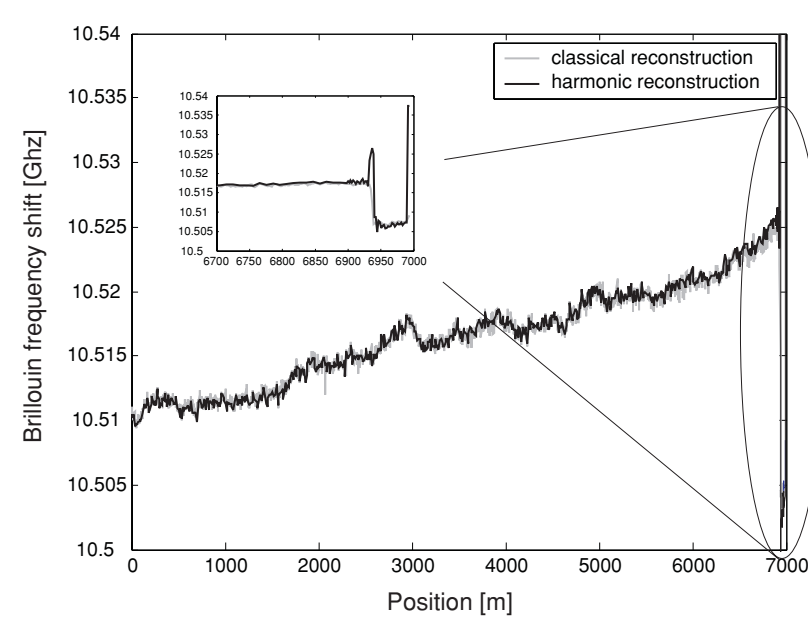

Figure 10. Reconstruction of the Brillouin frequency shift profile along the series of a $7 \mathrm{~km}$ long fibre and a $50 \mathrm{~m}$ long fibre by processing BOTDA loss measurements using the classical approach (grey line) and the harmonic approach (black line). The input optical powers are $P_{\mathrm{P} 0}=35 \mathrm{~mW}$ and $P_{\mathrm{CWL}}=200 \mu \mathrm{W}$.

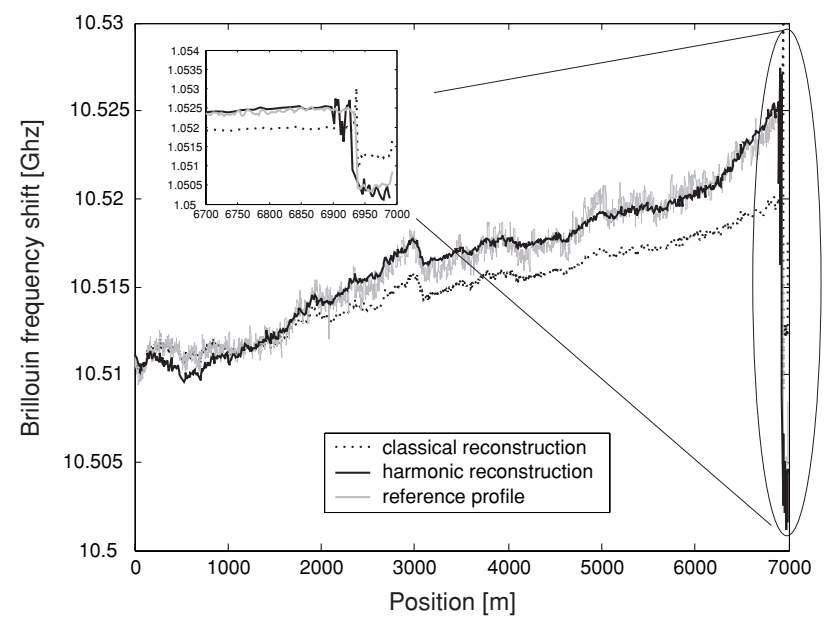

Figure 11. Reconstruction of the Brillouin frequency shift profile along the series of a $7 \mathrm{~km}$ long fibre and a $50 \mathrm{~m}$ long fibre by processing BOTDA loss measurements using the classical approach (dotted line) and the harmonic approach (black line), compared to the reference profile (grey line). The input optical powers are $P_{\mathrm{P} 0}=35 \mathrm{~mW}$ and $P_{\mathrm{CWL}}=4700 \mu \mathrm{W}$.

figure 10. As can be seen, there is an excellent agreement between the two reconstructions, as expected when considering the case $P_{\mathrm{CWL}}=200 \mu \mathrm{W}$. The next case refers to the measurement carried out in the Brillouin loss process with $P_{\mathrm{CWL}}=4700 \mu \mathrm{W}$. Results are shown in figure 11 . The capability of the harmonic reconstruction technique to compensate for nonlocal effects is demonstrated even in the correspondence of the last $50 \mathrm{~m}$ long region, as can be seen from the inset in figure 11.

\section{Conclusions}

The validity of a novel algorithm for temperature/strain profile reconstructions for BOTDA systems has been experimentally demonstrated. Based on a multidimensional minimization, it allows the reconstruction of the unknown profile along the fibre through its expansion in harmonic functions. A set of experimental tests demonstrated the capability of the algorithm to compensate for nonlocal effects suffered by the classical spectroscopic approach when dealing with sensing lengths of several kilometres, allowing the use of higher probe powers without the disadvantage of leading to unacceptable systematic errors. The computational time grows more than linearly with the number of harmonic components to be searched for, so it strongly depends on the profile to be reconstructed. However, any of the reconstructions shown in this paper required a number of harmonics below 20, corresponding to a processing time of the order of $10 \mathrm{~min}$, on an ordinary PC. Such a computational time, while being widely acceptable for distributed static measurements, could be significantly reduced by optimizing both the hardware and the software implementing the algorithm.

The proposed technique is specially intended for longrange distributed measurements (above $1 \mathrm{~km}$ ). For the reconstruction algorithm to work properly, a preliminary accurate knowledge of some sensing fibre features (attenuation, Brillouin gain spectrum) is needed. However, such a characterization of the sensing fibre has to be performed only once, and it can be achieved by using the same BOTDA equipment employed for the measurements.

\section{References}

[1] Chraplyvy A R 1990 Limitations on lightwave communications imposed by optical-fibre nonlinearities J. Lightwave Technol. 8 1548-57

[2] Horiguchi T and Tateda M 1989 Optical-fibre-attenuation investigation using stimulated Brillouin scattering between a pulse and a continuous wave Opt. Lett. 14 408-10

[3] Horiguchi T and Tateda M 1989 BOTDA—nondestructive measurement of single-mode optical fiber attenuation characteristics using Brillouin interaction: theory J. Lightwave Technol. 7 1170-6

[4] DeMerchant M D, Brown A W, Bao X and Bremmer T 2000 Signal processing for a high-spatial-resolution distributed sensor Opt. Eng. 39 1632-5

[5] Horiguchi T, Shimizu K, Kurashima T, Tateda M and Koyamada Y 1995 Development of a distributed sensing technique using Brillouin scattering J. Lightwave Technol. 13 1296-302

[6] Geinitz E, Jetshke S, Röpke U, Schröter S, Willsch R and Bartelt H 1999 The influence of pulse amplification on distributed fiber-optic Brillouin sensing and a method to compensate for systematic errors Meas. Sci. Technol. 10 112-6

[7] Bernini R, Minardo A and Zeni L 2002 Reconstruction technique for stimulated Brillouin scattering distributed fiber optic sensors Opt. Eng. 41 2186-94

[8] Agrawal G P 1989 Nonlinear Fiber Optics (Boston, MA: Academic) p 372

[9] Bao X, Dhiliwayo J, Heron N, Webb D J and Jackson D A 1995 Experimental and theoretical studies on a distributed temperature sensor based on Brillouin scattering J. Lightwave Technol. 13 1340-8

[10] van Deventer M O and Boot A J 1994 Polarizations properties of stimulated Brillouin scattering in single-mode fibers J. Lightwave Technol. 12 585-9

[11] Garus D, Gogolla T, Krebber K and Schliep F 1997 Brillouin optical-fiber frequency-domain analysis for distributed 
temperature and strain measurements J. Lightwave Technol. $15654-62$

[12] Tsun T O, Wada A, Sakai T and Yamauchi R 1992 Novel method using white spectral probe signals to measure Brillouin gain spectra of pure silica core fibers Electron. Lett. 28 247-9
[13] Nikles M, Thevenaz L and Robert P A 1997 Brillouin gain spectrum characterization in single-mode optical-fibers J. Lightwave Technol. 15 1842-51

[14] Zeni L, Bernini R and Pierri R 1999 Reconstruction of doping profiles in semiconductor materials using optical tomography Solid-State Electron. $\mathbf{4 3} 761-9$ 